Adenocarcinoma of prostate with mucinous differentiation arising in the male urethra is extremely rare, with only 21 cases reported in the previous literature. A diagnosis of mucin-producing urothelial carcinoma of the prostate is based on the pathology, immunohistochemistry, and clinical examination by excluding the secondary adenocarcinoma of the prostate. We present a case of unexpected mucinous urothelial carcinoma of prostate with co-existing inverted papilloma of bladder in a 57-year-old man. The patient underwent transurethral resection of the prostate (TURP) and transurethral resection of a bladder tumour (TUR-Bt), and the pathologic result showed mucinous prostate carcinoma and bladder inverted papilloma. Immunohistological stain was negative for prostate-specific antigen (PSA), prostate-specific acid phosphatase (PSAP), and $P 63$, but positive for cytokeratin 7 (CK 7), CK 20, clone $34 \beta E 12$ and P504S. A complete endoscopic examination was performed to exclude the secondary adenocarcinoma of prostate. This case illustrates the clinical and pathological features of a rare and unexpected mucin-producing urothelial carcinoma of prostate in a bladder neoplasm patient.

Key words: mucinous adenocarcinoma urothelial carcinoma, prostate, bladder neoplasm, immunohistochemistry.

Contemp Oncol (Pozn) 2017; 21 (2): 184-187 DOI: https://doi.org/10.5114/wo.2017.68629

\section{Co-existence of mucin-producing urothelial-type adenocarcinoma of the prostate and inverted papilloma of the bladder}

\author{
Xiao-Nan Mu', Si-Jun Wang ${ }^{2}$, Zhi-Gang Sun ${ }^{3}$, Min Zhang ${ }^{4}$, Zhe Li ${ }^{5}$, \\ Long-Yang Zhang ${ }^{2}$
}

1Department of Concerning Foreign Affairs, Jinan Central Hospital Affiliated to Shandong University, Jinan, P.R. China

2Department of Urology, Jinan Central Hospital Affiliated to Shandong University, Jinan, P.R. China

${ }^{3}$ Department of Thoracic Surgery, Jinan Center Hospital Affiliated to Shandong University, Shandong University, Jinan, P.R. China

${ }^{4}$ Department of Dermatology, Jinan Center Hospital Affiliated to Shandong University, Jinan, P.R. China

${ }^{5}$ Department of Examination Center, Jinan Center Hospital Affiliated to Shandong University, Jinan, P.R. China

\section{Introduction}

Adenocarcinoma of prostate with mucinous differentiation arising in the male urethra is extremely rare. To our knowledge, only 21 cases were reported in the previous literature [1-5]. Here we report a case of unsuspected urothelial-type adenocarcinoma of the prostate in a patient with inverted papilloma of the bladder. The initial cystoscopy in another hospital showed bladder tumour and benign prostate hyperplasia (BPH). After TUR-Bt and TURP, the histological result showed mucinous prostate carcinoma and bladder inverted papilloma. We report the clinical features and immunohistochemistry of this case.

\section{Case report}

A 57-year-old man presented with mucusuria and dysuresia relieved by ejaculation was hospitalized in May 2011. Ultrasonography and cystoscopy in another hospital showed co-existence of a bladder tumour about $0.7 \mathrm{~cm}$ in the bladder neck without biopsy and BPH. Digital rectal examination showed no nodule in the prostate. His serum PSA was $0.7 \mathrm{ng} / \mathrm{ml}$. He received TUR-Bt and TURP. The histological result showed that inverted papilloma of bladder and mucinous adenocarcinoma of the prostate. In order to exclude the secondary mucinous adenocarcinoma of the prostate, a complete endoscopic examination was performed, and bone scan showed no metastatic tumour. Immunohistology was negative for PSA, PSAP and P63, but positive for CK7, CK20, $\alpha$-methylacyl-coenzyme A racemase (AMACR, P504S) and high-molecular-weight-cytokeratin (HMWCK, clone 34ßE12). This patient received radical prostatectomy and has survived until now. We found that the prostatic urethra was full of white flocc material and a bladder tumour of $0.7 \mathrm{~cm}$ positioned at 5 o'clock of the bladder neck through the resectoscope, and a significant amount of tremelloid material coming from the prostate into the prostatic urethra during the procedure of TURP. A hematoxylin and eosin (H\&E) stained section showed that the bladder neoplasia was inverted papilloma (Fig. 1) and mucinous adenocarcinoma in the prostate specimen. In the prostate specimen, the tumor cell were tall and columnar and with heteromorphism nucleoli. The specimens showed the adenocarcinoma with cribriform, nidulant architecture in the lakes of extracellular mucin (Fig. 2-7). The study was approved by the Medical Ethics Committee for human studies of Shandong University of Medical Sciences. 


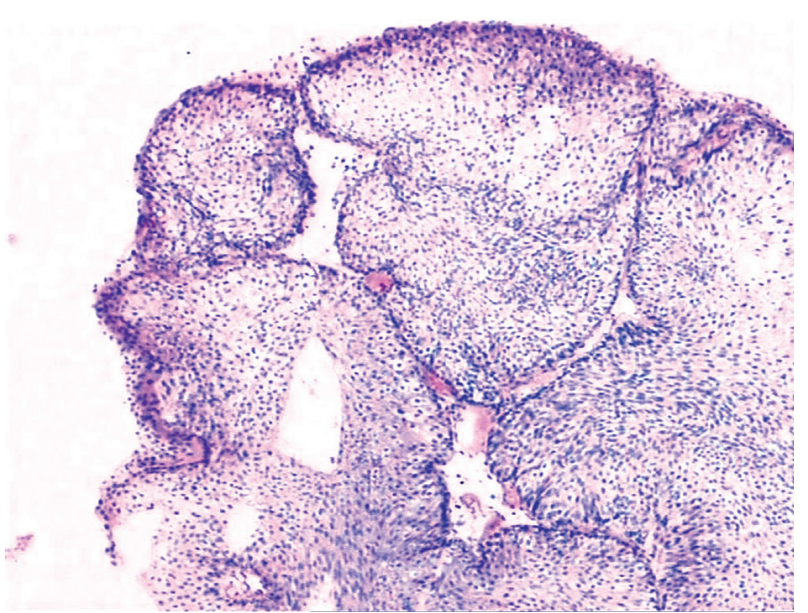

Fig. 1. Bladder inverted papilloma (H\&E stain, magnification 100x)

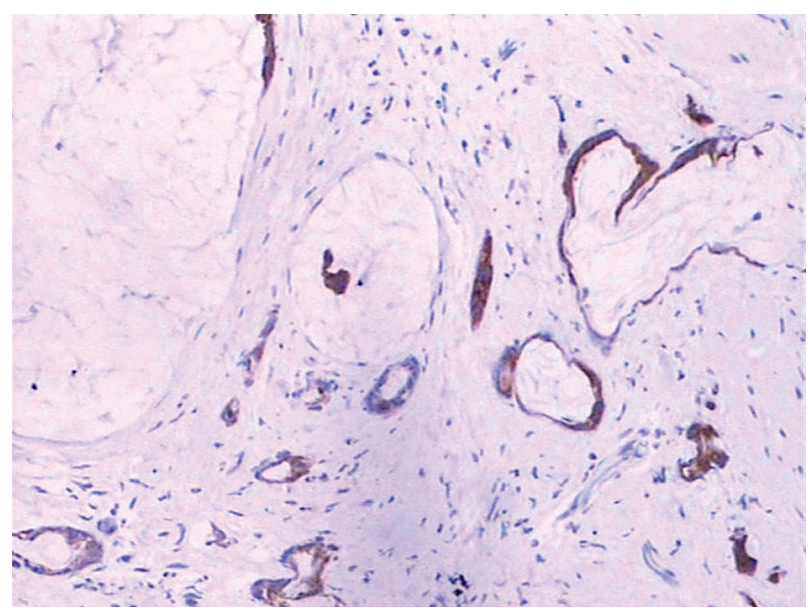

Fig. 3. Diffusely positive staining for CK7 (magnification 100x)

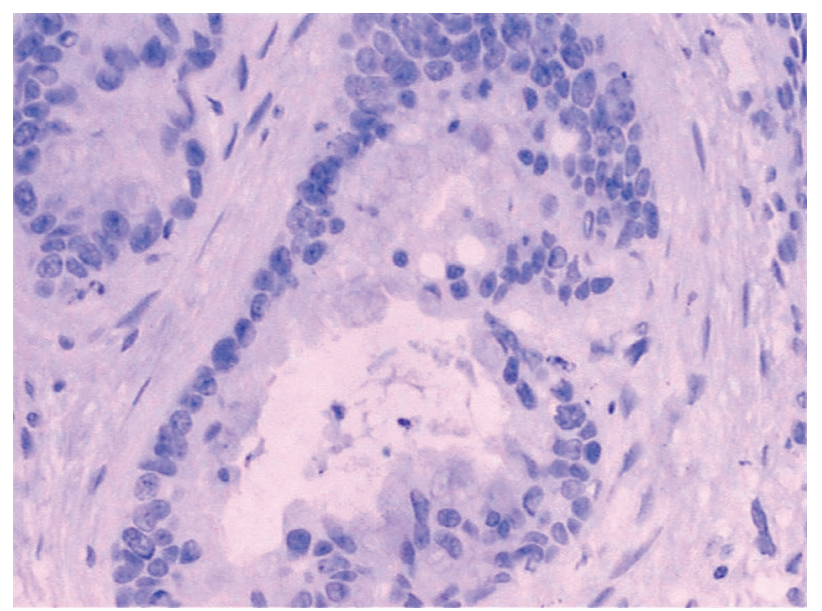

Fig. 5. Complete negative staining for p63 (magnification 200x)

\section{Discussion}

The incidence of unsuspected prostate cancer in bladder neoplasia patients in Asia was reported to be 4\% [6] [6], and the incidence in western countries was from $27 \%$ to $46 \%$ [7-12]. The primary adenocarcinoma arising in the

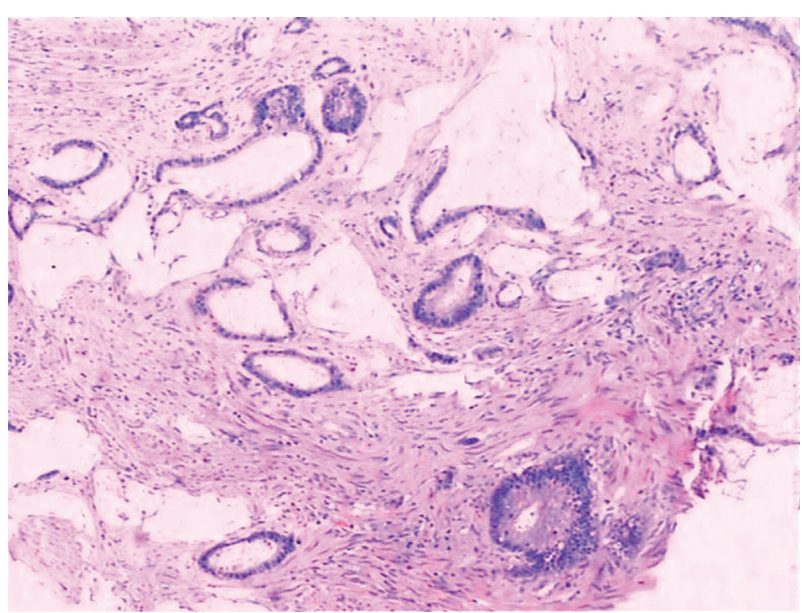

Fig. 2. Urothelial-type adenocarcinoma of prostate (H\&E stain, magnification $40 x$

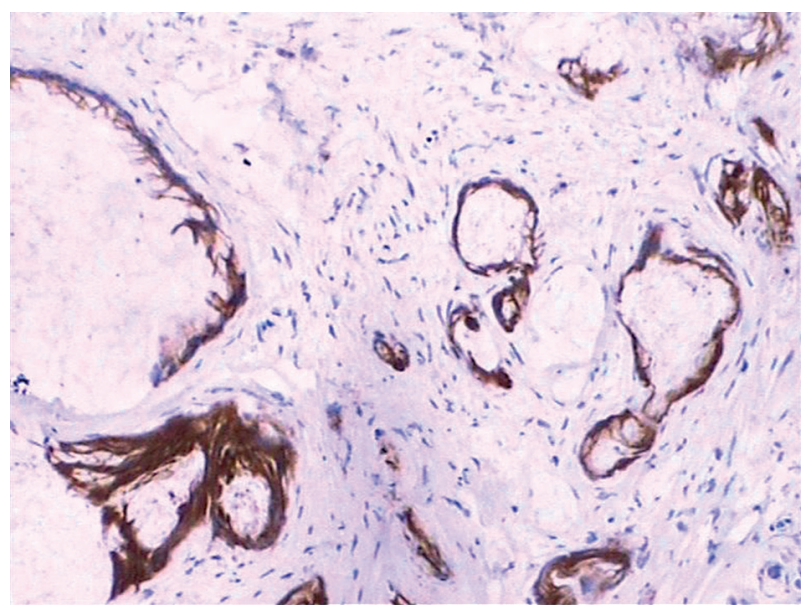

Fig. 4. Diffusely positive staining for CK 20 (magnification 100x)

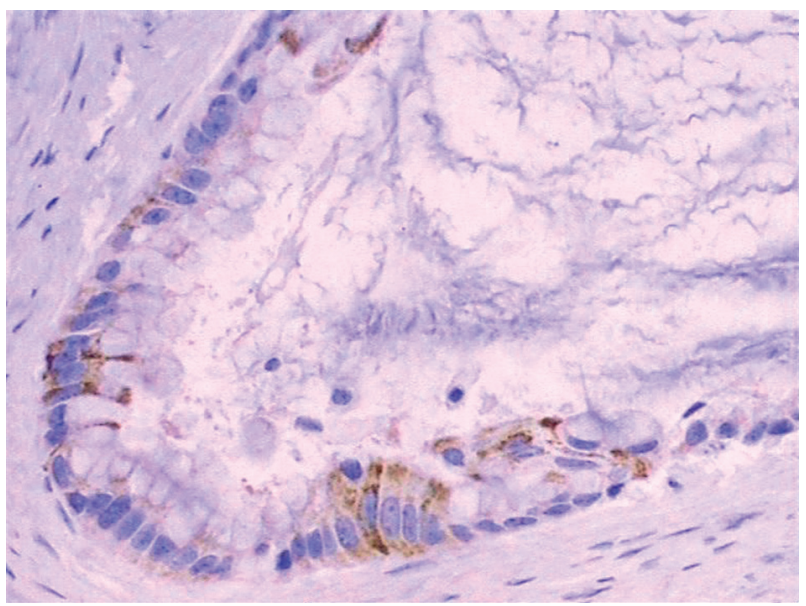

Fig. 6. Weak positive staining for P504S (magnification 200x)

male urethra involving the prostate is extremely rare, with only 21 cases reported in the previous literature, and there is no paper report about unsuspected urothelial-type adenocarcinoma of the prostate in a patient with inverted papilloma of the bladder. 


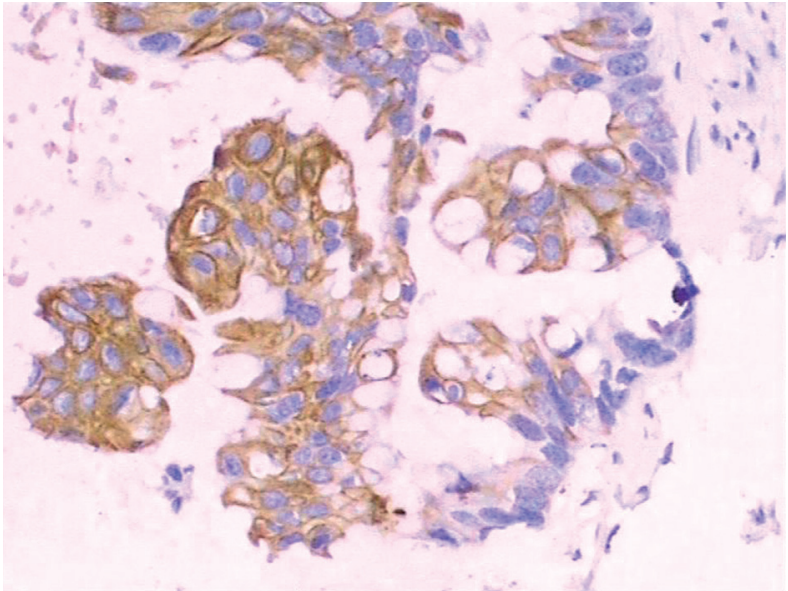

Fig. 7. Diffusely positive staining for 34ßE12 (magnification 200x)

Urinary obstruction and mucusuria are the most common presentations of the patients with urothelial-type adenocarcinoma of the prostate [4], which were the main presented signs of the patient in the present study. However, dysuresia and mucusuria in the patient of our study could be relieved significantly after ejaculation. We could not ideally explain this manifestation until we performed the operation, during which we found a lot of white flocc material in the prostatic urethra. We then concluded that the white flocc material in the prostatic urethra was discharged by ejaculation, and this explained the relief of dysuresia and mucusuria.

Accurate diagnosis of urothelial-type adenocarcinoma of the prostate is very important. The patient in our study was subjected to expensive and invasive examinations to rule out the primary tumour in the gastrointestinal tract. If the correct diagnosis had been established, it would be possible for the patient to receive the right treatment with lower cost, less invasive examination, and shorter hospitalisation. The differential diagnosis for urothelial-type adenocarcinoma of the prostate includes conventional prostatic adenocarcinoma with mucin production and metastatic colonic adenocarcinoma. The diagnosis of mucinous adenocarcinoma of the prostate includes a total tumour volume at least $25 \%$ mucinous and single or clustered tumour cells floating in mucin lakes [13]. It is crucial that the tumour cells express PSA and PSAP [13, 14].

It is impossible to distinguish urothelial-type adenocarcinoma of the prostate from secondary colonic adenocarcinoma only based on H\&E cytology, especially using prostate needle biopsies and/or TURP specimens [2]. Immunohistochemistry is crucial to establish a prescise diagnosis [3, 15]. CK7, CK20, and HMWCK (clone 34ßE12) have been utilized as potential urothelial markers [16-18]. CK7 is typically positive in carcinoma $[15,19,20]$ and negative in colonic carcinoma [21], but CK20 and HMWCK (clone 34ßE12) are less specific and sensitive than CK7 in urothelial-type carcinoma [18]. P63, which was studied as a basal cell marker in the diagnosis of prostate cancer [22, 23], and was expressed in $92 \%$ of urothelial carcinoma cases [19]. $\alpha$-methylacyl-coenzyme A racemase (AMACR), known as P504S, plays a role in the $\beta$-oxidation of branched-chain fatty acids and fatty acids derivatives [24], and it is typically positive in prostate carcinoma $[25,26]$. However, it has significant limitations as a useful immunohistochemical marker for prostate cancer, because AMACR expression is also identified in $18-58 \%$ of cases of nephrogenic adenoma $[27,28]$ and $4-21 \%$ of benign prostatic glands [26, 29]. In this case, the prostate cancer cells were completely negative for PSA, PSAP, and p63, and weak positive for P504S; prostatic mucinous adenocarcinoma were excluded, and the tumour cells were diffusely positive for CK7, $\mathrm{CK} 20$, and 34ßE12. It was reported that the CK7+/CK20+ immunophenotype in colonic adenocarcinoma was identified from $5.0 \%$ to $15.3 \%$ [30-32], the metastatic colonic adenocarcinoma could not be excluded. But this patient had a negative result for gastrointestinal tract examination, and we conclude that the prostatic adenocarcinoma cell of this case originated in the prostatic urethra.

It is difficult to predict their behaviour because of the rarity of these tumours. Only 21 cases of such urothelial-type adenocarcinoma of prostate were reported in the previous literature. More data about the prognosis should be collected to predict its behaviour.

In conclusion, we have described one case of unsuspected urothelial-type adenocarcinoma of the prostate in a patient with inverted papilloma of the bladder. More data are needed to determine whether mucusuria and dysuresia relieved by ejaculation could be a characteristic complaint of patients with urothelial-type adenocarcinoma of the prostate. The primary prostatic adenocarcinoma could not be distinguished from secondary colonic adenocarcinoma because of its striking morphological resemblance and overlapping immunohistochemical phenotype. More cases of urothelial-type adenocarcinoma of the prostate are required to better determine its clinical and pathological features.

The authors would like to thank Shan Dong University for their cooperation.

The authors declare no conflict of interest.

\section{References}

1. Tran KP, Epstein JI. Mucinous adenocarcinoma of urinary bladder type arising from the prostatic urethra. Distinction from mucinous adenocarcinoma of the prostate. Am J Surg Pathol 1996; 20: 1346 50.

2. Curtis MW, Evans AJ, Srigley JR. Mucin-producing urothelial-type adenocarcinoma of prostate: report of two cases of a rare and diagnostically challenging entity. Mod Pathol 2005; 18: 585-90.

3. Adley BP, Maxwell K, Dalton DP, Yang XJ. Urothelial-type adenocarcinoma of the prostate mimicking metastatic colorectal adenocarcinoma. Int Braz J Urol 2006; 32: 681-7.

4. Osunkoya AO, Epstein J. Primary mucin-producing urothelial-type adenocarcinoma of prostate: report of 15 cases. Am J Surg Pathol 2007; 31: 1323-9.

5. Ortiz-Rey JA, Dos Santos JE, Rodriguez-Castilla M, Alvarez C, Farina $L$. Mucinous urothelial-type adenocarcinoma of the prostate. Scand J Urol Nephrol 2004; 38: 256-7.

6. Lee SH, Chang PL, Chen SM, et al. Synchronous primary carcinomas of the bladder and prostate. Asian J Androl 2006; 8: 357-9. 
7. Montie JE, Wood DP Jr, Pontes JE, Boyett JM, Levin HS. Adenocarcinoma of the prostate in cystoprostatectomy specimens removed for bladder cancer. Cancer 1989; 63: 381-5.

8. Pritchett TR, Moreno J, Warner NE, et al. Unsuspected prostatic adenocarcinoma in patients who have undergone radical cysto prostatectomy for transitional cell carcinoma of the bladder. J Urol 1988; 139: 1214-6

9. Romero FR, de Castro MG, Andriolo Junior A, de Meneses AH, Fernandes RC, Perez MD. Coexistence of prostate neoplasia in patients undergoing radical cystoprostatectomy due to vesical neoplasia. Int Braz J Urol 2004; 30: 296-301.

10. Abbas F, Hochberg D, Civantos F, Soloway M. Incidental prostatic adenocarcinoma in patients undergoing radical cystoprostatectomy for bladder cancer. Eur Urol 1996; 30: 322-326.

11. Moutzouris G, Barbatis C, Plastiras D, et al. Incidence and histolog ical findings of unsuspected prostatic adenocarcinoma in radical cystoprostatectomy for transitional cell carcinoma of the bladder. Scand J Urol Nephrol 1999; 33: 27-30.

12. Kabalin JN, McNeal JE, Price HM, Freiha FS, Stamey TA. Unsuspect ed adenocarcinoma of the prostate in patients undergoing cysto prostatectomy for other causes: incidence, histology and morphometric observations. J Urol 1989; 141: 1091-1094.

13. Ro JY, Grignon DJ, Ayala AG, Fernandez PL, Ordonez NG, Wishnow KI. Mucinous adenocarcinoma of the prostate: histochemical and immunohistochemical studies. Hum Pathol 1990; 21: 593-600.

14. Epstein JI, Lieberman PH. Mucinous adenocarcinoma of the pros tate gland. Am J Surg Pathol 1985; 9: 299-308.

15. Wang NP, Zee SZ, RJ, Bacchi CE, Gown AM. Coordinate expression of cytokeratins 7 and 20 defines unique subsets of carcinomas. Appl Immunohistochem 1995; 3: 99-107.

16. Bassily NH, Vallorosi CJ, Akdas G, Montie JE, Rubin MA. Coordinate expression of cytokeratins 7 and 20 in prostate adenocarcinoma and bladder urothelial carcinoma. Am J Clin Pathol 2000; 113: 383-8.

17. Varma M, Morgan M, Amin MB, Wozniak S, Jasani B. High molec ular weight cytokeratin antibody (clone 34betaE12): a sensitive marker for differentiation of high-grade invasive urothelial carci noma from prostate cancer. Histopathology 2003; 42: 167-72.

18. Genega EM, Hutchinson B, Reuter VE, Gaudin PB. Immunophenotype of high-grade prostatic adenocarcinoma and urothelial carcinoma. Mod Pathol 2000; 13: 1186-91.

19. Kunju LP, Mehra R, Snyder M, Shah RB. Prostate-specific antigen, high-molecular-weight cytokeratin (clone 34betaE12), and/or p63: an optimal immunohistochemical panel to distinguish poorly differentiated prostate adenocarcinoma from urothelial carcinoma. Am J Clin Pathol 2006; 125: 675-81.

20. Nakamura Y, Orikasa K, Fujishima F, et al. A case of villous adeno ma of the urinary bladder with tubulovillous architecture: characterization by immunohistochemical analysis. Pol J Pathol 2011; 62: 179-82.

21. Berezowski K, Stastny JF, Kornstein MJ. Cytokeratins 7 and 20 and carcinoembryonic antigen in ovarian and colonic carcinoma. Mod Pathol 1996; 9: 426-9.

22. Shah RB, Zhou M, LeBlanc M, Snyder M, Rubin MA. Comparison of the basal cell-specific markers, 34betaE12 and p63, in the diagnosis of prostate cancer. Am J Surg Pathol 2002; 26: 1161-8.

23. Zhou M, Shah R, Shen R, Rubin MA. Basal cell cocktail (34betaE12 + p63) improves the detection of prostate basal cells. Am J Surg Pathol 2003; 27: 365-71.

24. Ferdinandusse S, Denis S, L IJ, et al. Subcellular localization and physiological role of alpha-methylacyl-CoA racemase. J Lipid Res 2000; 41: 1890-6

25. Jiang Z, Fanger GR, Woda BA, et al. Expression of alpha-methylacyl-CoA racemase (P504s) in various malignant neoplasms and normal tissues: astudy of 761 cases. Hum Pathol 2003; 34: 792-6.

26. Jiang Z, Woda BA, Rock KL, et al. P504S: a new molecular marker for the detection of prostate carcinoma. Am J Surg Pathol 2001; 25: 1397-404.

27. Gupta A, Wang HL, Policarpio-Nicolas ML, et al. Expression of alpha-methylacyl-coenzyme A racemase in nephrogenic adenoma. Am J Surg Pathol 2004; 28: 1224-9.
28. Allan CH, Epstein JI. Nephrogenic adenoma of the prostatic urethra: a mimicker of prostate adenocarcinoma. Am J Surg Pathol 2001; 25: 802-8.

29. Luo J, Zha S, Gage WR, et al. Alpha-methylacyl-CoA racemase: a new molecular marker for prostate cancer. Cancer Res 2002; 62: 2220-6.

30. Chu P, Wu E, Weiss LM. Cytokeratin 7 and cytokeratin 20 expression in epithelial neoplasms: a survey of 435 cases. Mod Pathol 2000; 13: $962-72$

31. Bayrak R, Yenidunya S, Haltas H. Cytokeratin 7 and cytokeratin 20 expression in colorectal adenocarcinomas. Pathol Res Pract 2011; 207: 156-60

32. Vang R, Gown AM, Wu LS, et al. Immunohistochemical expression of CDX2 in primary ovarian mucinous tumors and metastatic mucinous carcinomas involving the ovary: comparison with CK20 and correlation with coordinate expression of CK7. Mod Pathol 2006; 19: $1421-8$

\section{Address for correspondence}

\section{Si-Jun Wang}

Department of Urology

Jinan Central Hospital Affiliated to Shandong University

Jinan, 250013, P.R. China

e-mail:wsjmxn@126.com

\section{Zhi-Gang Sun}

Department of Thoracic Surgery

Jinan Center Hospital Affiliated to Shandong University

Shandong University

Jinan 250013, P.R. China

e-mail: sunszg@126.com

Submitted: 2.06.2013

Accepted: $\quad 24.11 .2013$ 